\title{
An adaptive two-level method for hypersingular integral equations in $\mathrm{R}^{3}$
}

\author{
Patrick Mund* $\quad$ Ernst P. Stephan*
}

(Received 7 August 2000)

\begin{abstract}
In this paper an a posteriori error estimate for hypersingular integral equations is derived by using hierarchical basis techniques. Based on the properties of a two-level additive Schwarz method easily computable local error indicators are obtained. An algorithm for adaptive error control which allows anisotropic refinements of the boundary elements is formulated and numerical results are included.
\end{abstract}

*Institut für Angewandte Mathematik, Universität Hannover, Welfengarten 1, 30167 Hannover, GERMANY. mailto:stephan@ifam.uni-hannover.de

${ }^{0}$ See http: //anziamj . austms . org. au/V42/CTAC99/Mund for this article and ancillary services, (C) Austral. Mathematical Soc. 2000. Published 27 Nov 2000. 


\section{Contents}

1 A stable two-level subspace decomposition

C1020

2 An a posteriori error estimate

C1026

3 Numerical results

C1028

References

C1032

\section{A stable two-level subspace decomposition}

In recent years adaptive hierarchical basis methods in the finite element method (FEM) [1, 2, 4] have become increasingly popular. This approach has meanwhile been also applied to the boundary element method (BEM) for weakly singular integral equations [8] and to the FEM/BEM coupling [7]. Here we extend it to hypersingular integral equations on surfaces.

We consider the hypersingular integral equation

$$
W v(x):=-\frac{1}{4 \pi} \frac{\partial}{\partial n_{x}} \int_{\Gamma} v(y) \frac{\partial}{\partial n_{y}} \frac{1}{|x-y|} d \sigma_{y}=f(x), \quad x \in \Gamma
$$

where $\Gamma$ is an open plane surface and integration has to be understood in the Hadamard sense. $W$ is a bijective mapping from $\tilde{H}^{1 / 2}(\Gamma)$ onto $H^{-1 / 2}(\Gamma)$ and 
the bilinear form $\langle W u, v\rangle$ for $u, v \in \tilde{H}^{1 / 2}(\Gamma)$ is symmetric and positive definite (cf. Costabel [3], Stephan [9]). Hence the unique solution $v_{N} \in S_{N} \subset \tilde{H}^{1 / 2}(\Gamma)$ of the Galerkin scheme

$$
\left\langle W v_{N}, \chi\right\rangle=\langle f, \chi\rangle \quad \forall \chi \in S_{N}
$$

converges quasi-optimally towards the exact solution $v \in \tilde{H}^{1 / 2}(\Gamma)$ of $(1)$. For standard Sobolev spaces $L^{2}(\Gamma)$ and $H_{0}^{1}(\Gamma)$ (which is the completion of $C_{0}^{\infty}$ within $\left.H^{1}(\Gamma)\right)$ the space $\tilde{H}^{1 / 2}(\Gamma)$ is an interpolation space between $L^{2}(\Gamma)$ and $H_{0}^{1}(\Gamma)$, and $H^{-1 / 2}(\Gamma)$ is the dual space of $\tilde{H}^{1 / 2}(\Gamma)$. The solution $u$ of $(1)$ for given $f \in H^{-1 / 2}(\Gamma)$ is the jump across $\Gamma$ of the solution of a Neumann problem for the Laplacian in $\mathrm{R}^{3} \backslash \bar{\Gamma}$, cf. [9].

In the following we present a two-level method for the $h$-version of the Galerkin scheme with bilinear continuous elements. For ease of presentation we consider $\Gamma=[-1,1]^{2}$.

Let $\gamma_{l}(0 \leq l \leq L)$ be a uniform partition of $\Gamma$ into squares of side length $h_{l}=2^{-l}$ and let

$$
\begin{aligned}
X_{l} & =\left\{u \in \mathcal{C}^{0}(\Gamma): u \text { piecewise bilinear w.r.t. } \gamma_{l} \text { and } u_{\mid \partial \Gamma}=0\right\}(0 \leq l \leq L) \\
X_{l}^{0} & =\left\{u \in X_{l}: u=0 \text { on the nodes of } \gamma_{l-1}\right\} \quad(1 \leq l \leq L) .
\end{aligned}
$$

Let $b_{l, i}\left(0 \leq i \leq n_{l}\right)$ be a piecewise bilinear function with value one at an interior node of $\gamma_{l}$ (not belonging to $\gamma_{l-1}$ ) and with zero value in all other nodes of $\gamma_{l}$. Let $X_{L, i}^{0}=\operatorname{span}\left\{b_{L, i}\right\}$. We have the following two-level 
decomposition of the space $X_{L}$ :

$$
X_{L}=X_{L-1} \oplus X_{L, 1}^{0} \oplus \cdots \oplus X_{L, n_{L}}^{0}
$$

Let

$$
P_{(2)}^{L}=P_{L-1}+\sum_{i=1}^{n_{L}} P_{L, i}
$$

be the two-level additive Schwarz operator belonging to the subspace decomposition (3) and the bilinear form $\langle W \cdot, \cdot\rangle$, i.e.

$$
\begin{aligned}
\left\langle W P_{L-1} \phi, \psi\right\rangle & =\langle W \phi, \psi\rangle \quad \forall \psi \in X_{L-1}, \phi \in X_{L}, \\
\left\langle W P_{L, i} \phi, \psi\right\rangle & =\langle W \phi, \psi\rangle \quad \forall \psi \in X_{L, i}^{0}, \phi \in X_{L} .
\end{aligned}
$$

Then there holds

Theorem 1 There exist constants $c_{1}, c_{2}>0$, independent of $L$, such that

$$
c_{1}\langle W u, u\rangle \leq\left\langle W P_{(2)}^{L} u, u\right\rangle \leq c_{2}\langle W u, u\rangle \quad \forall u \in X_{L} .
$$

The proof of Theorem 1 is based on the following lemmas where always $u \in X_{L}$ arbitrary with

$$
u=u_{L-1}+\sum_{i=1}^{n_{L}} u_{L, i}
$$

where $u_{L-1} \in X_{L-1}$ and $u_{L, i} \in X_{L, i}^{0}$. Let $I_{L-1} u \in X_{L-1}$ be the bilinear interpolant of $u$ at the nodes of $\gamma_{L-1}$. 
Lemma 2 [6, Lemma 2.5, Lemma 3.10] There exists a constant c, independent of $L$ and $u$, such that

$$
\left\|u-I_{L-1} u\right\|_{L^{2}(\Gamma)} \leq c h_{L}\|u\|_{H^{1}(\Gamma)} .
$$

Furthermore for any $s \in[0,1]$ there exists a constant $c=c(s)>0$ such that

$$
\left\|I_{L-1} u\right\|_{\tilde{H}^{s}(\Gamma)} \leq c\|u\|_{\tilde{H}^{s}(\Gamma)} \quad \text { for any } u \in X_{L} .
$$

Lemma 3 [6, Lemma 3.12] Let $\Gamma_{i}^{L} \in \gamma_{L} \quad$ be a square with vertices $x_{k}$ $(1 \leq k \leq 4)$. Let $v, w$ be bilinear functions on $\Gamma_{i}^{L}$ with $v\left(x_{1}\right)=w\left(x_{1}\right)$ and $w\left(x_{2}\right)=w\left(x_{3}\right)=w\left(x_{4}\right)=0$. Then there holds

$$
\|w\|_{L^{2}\left(\Gamma_{i}^{L}\right)} \leq \frac{4}{3}\|v\|_{L^{2}\left(\Gamma_{i}^{L}\right)}
$$

Lemma 4 [5] Let $\left\{\Gamma_{i}, i=1, \ldots, N\right\}$ be a finite covering of $\Gamma$ with rectangles $\Gamma_{i}$ and covering constant $\sigma \in \mathrm{N}$, i.e. we can colour $\left\{\Gamma_{i}, i=1, \ldots, N\right\}$ by at most $\sigma$ different colours such that subdomains with same colour are disjoint. Let $\phi=\sum_{i=1}^{N} \phi_{i} \in \tilde{H}^{s}(\Gamma)$ for $s \in \mathrm{R}$ with $\phi_{i} \in \tilde{H}^{s}\left(\Gamma_{i}\right)$. Then there holds

$$
\|\phi\|_{\tilde{H}^{s}(\Gamma)}^{2} \leq \sigma \sum_{i=1}^{N}\left\|\phi_{i}\right\|_{\tilde{H}^{s}\left(\Gamma_{i}\right)}^{2}
$$


Proof: (of Theorem 1) We show that there exist constants $c_{1}, c_{2}>0$ such that

$$
\frac{1}{c_{2}}\|u\|_{\tilde{H}^{1 / 2}(\Gamma)}^{2} \leq\left\|u_{L-1}\right\|_{\tilde{H}^{1 / 2}(\Gamma)}^{2}+\sum_{i=1}^{n_{L}}\left\|u_{L, i}\right\|_{\tilde{H}^{1 / 2}(\Gamma)}^{2} \leq \frac{1}{c_{1}}\|u\|_{\tilde{H}^{1 / 2}(\Gamma)}^{2} .
$$

The left inequality follows directly from Lemma 4 with $c_{2}=9$ since to any $x \in \Gamma$ there belong at most 8 different $b_{L, i}$ 's with $x \in \operatorname{supp} b_{L, i}\left(1 \leq i \leq n_{L}\right)$ and since

$$
\left\|u_{L, i}\right\|_{\tilde{H}^{1 / 2}(\Gamma)}=\left\|u_{L, i}\right\|_{\tilde{H}^{1 / 2}\left(\operatorname{Supp}_{L, i}\right)}
$$

It remains to show the right inequality in (8). Since $u_{L-1}=I_{L-1} u$ Lemma 2 implies

$$
\left\|u_{L-1}\right\|_{\tilde{H}^{1 / 2}(\Gamma)} \leq c\|u\|_{\tilde{H}^{1 / 2}(\Gamma)}
$$

Let $\left\{x_{i}\right\}_{i=1}^{n_{L}}$ denote the set of nodes in $\gamma_{L}$ which do not belong to $\gamma_{L-1}$. We decompose the index set $\left\{1,2, \ldots, n_{L}\right\}=M_{1} \cup M_{2} \cup M_{3}$ into 3 disjoint sets $M_{k}$ such that two indices $i, j \in\left\{1,2, \ldots, n_{L}\right\}$ belong to the same set $M_{k}$ if $\left|x_{i}-x_{j}\right|$ is an integer multiple of $2 h_{L}$. The sets $M_{k}$ are uniquely determined (up to permutation) (cf. Fig. 1). For $k \in\{1,2,3\}$ the nodes $\left\{x_{i}\right\}_{i \in M_{k}}$ are just the nodes of the coarse grid $\gamma_{L-1}$ shifted by $h_{L}$ in the $x_{1}$ - and/or $x_{2}$-direction. There holds for $k \in\{1,2,3\}$

$$
\operatorname{meas}\left(\operatorname{supp} b_{L, i} \cap \operatorname{supp} b_{L, j}\right)=0 \quad \forall i, j \in M_{k}, i \neq j
$$




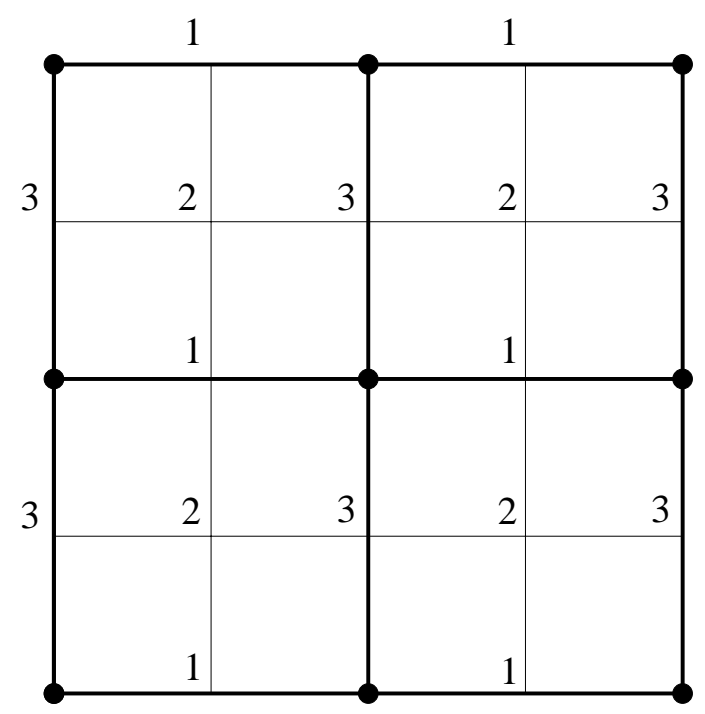

Figure 1: Decomposition of the nodes $\gamma_{L}$ into disjoint subsets: Number $k \in\{1,2,3\}$ of the node $x$ means $x \in M_{k}$. The nodes of the coarse mesh $\gamma_{L-1}$ are marked with 
with the Lebesgue-measure meas $(\cdot)$. Hence

$$
\sum_{i \in M_{k}}\left\|u_{L, i}\right\|_{0}^{2}=\left\|\sum_{i \in M_{k}} u_{L, i}\right\|_{0}^{2} \leq \frac{16}{9}\|\tilde{u}\|_{0}^{2}
$$

where $\tilde{u}=u-u_{L-1}$ and $\|\cdot\|_{s}=\|\cdot\|_{\tilde{H}^{s}(\Gamma)}, s \in \mathbb{R}$.

Here the last inequality follows from Lemma 3 since $w=\sum_{i \in M_{k}} u_{L, i}$ and $\tilde{u}$ coincide at one node of each element in $\gamma_{L}$ and $w$ vanishes in all the other nodes. With the standard inverse inequality for finite elements and (10) and (6) we have

$$
\begin{array}{rlrl}
\sum_{i=1}^{n_{L}}\left\|u_{L, i}\right\|_{1 / 2}^{2} & \leq \quad c h_{L}^{-1} \sum_{i=1}^{n_{L}}\left\|u_{L, i}\right\|_{0}^{2} & =c h_{L}^{-1} \sum_{k=1}^{3} \sum_{i \in M_{k}}\left\|u_{L, i}\right\|_{0}^{2} \\
\leq & c^{\prime} h_{L}^{-1}\|\tilde{u}\|_{0}^{2} & & \leq c^{\prime} h_{L}^{-1}\left\|u-I_{L-1} u\right\|_{0}^{2} \\
\leq & c h_{L}\|u\|_{1}^{2} & \leq c\|u\|_{1 / 2}^{2} .
\end{array}
$$

Together with (9) this implies the right inequality in (8). Thus the proof is complete due to the equivalence of the norms $\|u\|_{1 / 2}$ and $\langle W u, u\rangle^{1 / 2}$.

\section{An a posteriori error estimate}

Next we need the saturation assumption: $\left(A_{h}\right)$ There exist constants $k_{0} \in \mathrm{N}$ and $0<\rho<1$ such that

$$
\left\|v-v_{k+1}\right\|_{1 / 2} \leq \rho\left\|v-v_{k}\right\|_{1 / 2} \quad \forall k \geq k_{0}
$$


where $v_{k+1}$ denotes the Galerkin solution on the level $k+1$ and $v$ the exact solution of (1).

Theorem 5 Suppose $\left(A_{h}\right)$ holds. Then there exist constants $c_{1}, c_{2}>0$ such that there holds for $k \geq k_{0}$

$$
c_{1} \sum_{j=1}^{n_{L}} \eta_{L, j}^{2} \leq\left\|v-v_{L-1}\right\|_{1 / 2}^{2}=c_{2} \sum_{j=1}^{n_{L}} \eta_{L, j}^{2}
$$

with

$$
\eta_{L, j}=\frac{\left|\left\langle f-W v_{L-1}, b_{L, j}\right\rangle\right|}{\left\langle W b_{L-1}, b_{L, j}\right\rangle^{1 / 2}} \quad\left(j=1,2, \ldots, n_{L}\right)
$$

Proof: The saturation assumption $\left(A_{h}\right)$ yields the equivalence of norms

$$
\left\|v_{L}-v_{L-1}\right\|_{1 / 2} \sim\left\|v-v_{L-1}\right\|_{1 / 2} .
$$

Due to Theorem 1 we have

$$
\begin{array}{r}
c_{1}\left\|v_{L}-v_{L-1}\right\|_{1 / 2}^{2} \leq\left\|P_{L-1}\left(v_{L}-v_{L-1}\right)\right\|_{1 / 2}^{2}+\sum_{i=1}^{n_{L}}\left\|P_{L, i}\left(v_{L}-v_{L-1}\right)\right\|_{1 / 2}^{2} \\
\leq c_{2}\left\|v_{L}-v_{L-1}\right\|_{1 / 2}^{2} .
\end{array}
$$

Firstly, we observe that since $v_{L-1}$ and $v_{L}$ satisfy the Galerkin equation there holds for any $w \in X_{L-1}$

$$
\left\langle W P_{L-1} v_{L}, w\right\rangle=\left\langle W v_{L}, w\right\rangle=\langle f, w\rangle=\left\langle W v_{L-1}, w\right\rangle=\left\langle W P_{L-1} v_{L-1}, w\right\rangle .
$$


Hence

$$
\left\|P_{L-1}\left(v_{L}-v_{L-1}\right)\right\|_{1 / 2}^{2}=0 .
$$

The error indicator $\eta_{L, j}$ in (12) is obtained by solving a linear problem in the space $X_{L, j}^{0}$. The function $v_{L, j}=P_{L, j}\left(v_{L}-v_{L-1}\right) \in X_{L, j}^{0}$ solves for any $v \in X_{L, j}^{0}$

$$
\left\langle W v_{L, j}, v\right\rangle=\left\langle f-W v_{L-1}, v\right\rangle
$$

Hence firstly one solves (13) for $1 \leq j \leq n_{L}$ and then one computes the terms $\eta_{L, j}=\left\langle W v_{L, j}, v_{L, j}\right\rangle^{1 / 2}$. Since $X_{L, j}^{0}=\operatorname{span}\left\{b_{L, j}\right\}$ is a one-dimensional space, we have $v_{L, j}=c b_{L, j}$ with coefficient

$$
c=\frac{\left\langle f-W v_{L-1}, b_{L, j}\right\rangle}{\left\langle W b_{L, j}, b_{L, j}\right\rangle} .
$$

Hence

$$
\eta_{L, j}=|c|\left\langle W b_{L, j}, b_{L, j}\right\rangle^{1 / 2} .
$$

\section{$3 \quad$ Numerical results}

Algorithm 3.1 (Adaptive multilevel algorithm) Let $\gamma_{0}$ denote an initial mesh on $\Gamma$ and $X_{0}$ the corresponding space of continuous bilinear functions. Furthermore let $0 \leq \theta \leq 1$ and $\delta>0$ be given. 
1. Compute the Galerkin solution $u_{k} \in X_{k}$ of (2).

2. Compute the error indicators $\eta_{k, j}, j=1, \ldots, n_{k}$ with (12).

3. Compute the row error indicators $\eta_{R, m}$ and the column error indicators $\eta_{C, n}$ as weighted sums of the single error indicators of one row and one column of the mesh, respectively. The weighting is done by dividing the quadratic mean of the local error indicators of a row or a column by the respective number of terms.

4. Compute $\eta_{\max }:=\max \left\{\eta_{R, m}, \eta_{C, n}\right\}$. Refine all elements of the $m^{\text {th }}$ row in $x_{1}$-direction if

$$
\eta_{R, m} \geq \theta \eta_{\max }
$$

refine all elements of the $n^{\text {th }}$ column in $x_{2}$-direction if

$$
\eta_{C, n} \geq \theta \eta_{\max }
$$

Here refining of the element with index $i$ in $x_{k}$-direction means halving the element in $x_{k}$-direction.

5. Thus obtain the space $X_{k+1}$. Check whether $\eta_{\max }<\delta$ is satisfied. Otherwise go to 1.

Remark 6 Due to the row and column error indicators the above refinement strategy secures the continuity of the trial functions. No hanging nodes are obtained. 
TABLE 1: Adaptive $h$-refinement with $\theta=0.6$.

\begin{tabular}{|c|c|c||c||c|c|c|}
\hline$L$ & $N_{L}$ & $E_{L}$ & $\eta_{L}$ & $\eta_{L} / E_{L}$ & $\kappa\left(B_{h} W_{h}\right)$ & $\kappa\left(W_{h}\right)$ \\
\hline 1 & 5 & 0.559700 & 0.335091 & 0.598697 & $1.24 \cdot 10^{0}$ & $1.24 \cdot 10^{0}$ \\
2 & 16 & 0.466277 & 0.313657 & 0.672684 & $4.86 \cdot 10^{0}$ & $2.36 \cdot 10^{0}$ \\
3 & 48 & 0.311299 & 0.201438 & 0.647088 & $1.39 \cdot 10^{1}$ & $3.74 \cdot 10^{0}$ \\
4 & 96 & 0.214100 & 0.130477 & 0.609423 & $1.65 \cdot 10^{1}$ & $6.99 \cdot 10^{0}$ \\
5 & 160 & 0.149149 & 0.085045 & 0.570201 & $1.77 \cdot 10^{1}$ & $1.37 \cdot 10^{1}$ \\
6 & 240 & 0.105005 & 0.057504 & 0.547632 & $1.80 \cdot 10^{1}$ & $2.86 \cdot 10^{1}$ \\
7 & 336 & 0.074666 & 0.041280 & 0.552871 & $1.83 \cdot 10^{1}$ & $6.13 \cdot 10^{1}$ \\
8 & 448 & 0.053782 & 0.032152 & 0.597816 & $1.84 \cdot 10^{1}$ & $1.34 \cdot 10^{2}$ \\
\hline
\end{tabular}

Next we choose the model problem $\Gamma$ to be the L-shaped surface piece in the $\left(x_{1}, x_{2}\right)$-plane with vertices $(0,0,0),(1,0,0),(1,-1,0),(-1,-1,0)$, $(-1,1,0),(0,1,0)$, and the right hand side $f=1$ in $(1)$. In Table 1 the results are presented for the adaptive refinement strategy. $N_{L}$ denotes the number of unknowns on level $L ; E_{L}$ the relative error in the energy norm; $\kappa\left(B_{h} W_{h}\right)$ and $\kappa\left(W_{h}\right)$ the condition numbers of the preconditioned and unpreconditioned Galerkin matrix of (2), respectively; $B_{h}$ the preconditioner according to the additive Schwarz operator in (4); $\eta_{L}$ the sum of the error indicators and $\eta_{L} / E_{L}$ the efficiency index of the algorithm. The sequence of corresponding mesh refinements is given in Figure 2. 

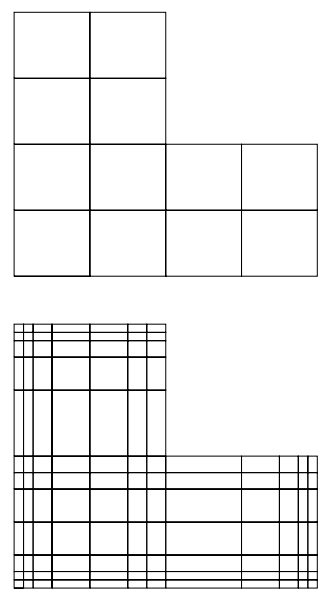
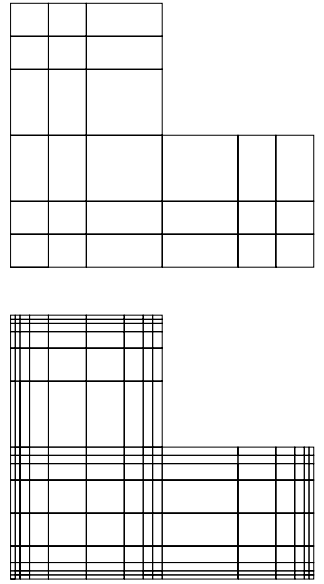
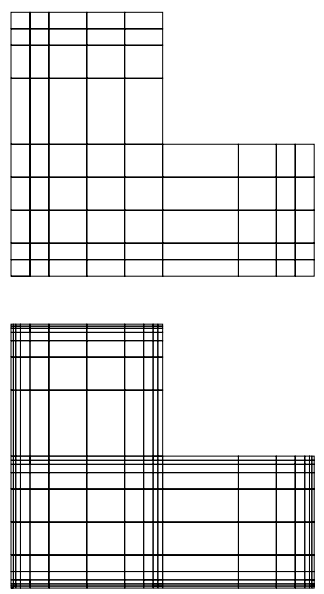

Figure 2: The sequence of refined meshes for $\theta=0.6$. 
Acknowledgments: This work was supported by the German Research Foundation (DFG) grants Ste 258/25-9 and Ste573/3-1. The authors would like to thank K.-W. Oertel for performing the numerical experiments and M. Maischak for providing his software package MAIPROGS.

\section{References}

[1] R.E. Bank and R.K. Smith. A posteriori error estimates based on hierarchical bases. SIAM J. Numer. Anal., 30:921-935, 1993. C1020

[2] F.A. Bornemann, B. Erdmann, and R. Kornhuber. A posteriori error estimates for elliptic problems in two and three space dimensions. SIAM J. Numer. Anal., 33:1188-1204, 1996. C1020

[3] Martin Costabel. Boundary integral operators on Lipschitz domains: Elementary results. SIAM J. Math. Anal., 19(3):613-626, 1988. C1021

[4] P. Deuflhard, P. Leinen, and H. Yserentant. Concepts of an adaptive hierarchical finite element code. Impact of Computing in Science and Engineering, 1:3-35, 1989. C1020

[5] Norbert Heuer. Additive schwarz methods for weakly singular integral equations in $\mathrm{R}^{3}$ - the $p$-version. In G. Wittum W. Hackbusch, editor, Boundary Elements: Implementation and Analysis of Advanced Algorithms, Proceedings of the 12th GAMM-Seminar, Kiel 1996, 
volume 54 of Notes on Numerical Fluid Mechanics, pages 126-135, Braunschweig, 1996. Vieweg. C1023

[6] Patrick Mund. Zwei-Level-Verfahren für Randintegralgleichungen mit Anwendungen auf die nichtlineare FEM-BEM-Kopplung. PhD thesis, Hannover, 1997. C1023, C1023

[7] Patrick Mund and Ernst Peter Stephan. An adaptive two-level method for the coupling of nonlinear fem-bem equations. SIAM J. Numer.Analysis, 36:1001-1021, 1999. C1020

[8] Patrick Mund, Ernst Peter Stephan, and Josha Weiße. Two-level methods for the single layer potential in $\mathrm{R}^{3}$. Computing, 60:243-266, 1998. C1020

[9] Ernst Peter Stephan. Boundary integral equations for screen problems in $\mathrm{R}^{3}$. Integral Equations and Operator Theory, 10:257-263, 1987. C1021, C1021 